\title{
The Study of PAHs Distribution in Underground Water in Southwest China
}

\author{
Junpeng Zhang, Shihua Qi, Hongfu Bao \\ Postdoctoral Workstation, \\ China Three Gorges Corporation, \\ Chengdu, China
}

\begin{abstract}
Polycyclic aromatic hydrocarbons have very strong toxicity, it has intended to must be controlled primary by U.S. Environmental Protection Agency and the European Union. In this study, we investigated the samples of groundwater in 7 provinces of southwest China. The main conclusion is: (i) we detected the pollutants in all samples in the study area; (ii) The migration of PAHs pollutants follow the global distillation model, and the contaminants of high latitudes was more than low latitudes; (iii) The PAHs were mainly comes from agricultural pollution and life pollution in the study area, and artificial source > natural source; (iv) The study area belongs to karst landform, organic environment is very fragile, it is more likely polluted by Persistent Organic Pollutants.
\end{abstract}

Keywords-polycyclic aromatic hydrocarbons; organic pollutants; karst

\section{INTRODUCTION}

PAHs (Polycyclic aromatic hydrocarbons) is come from incomplete combustion or high temperature cracking by product of organic matter. It widely exists in oil and coal, it has potential teratogenicity, carcinogenicity and genetic toxicity. With the increase of the benzene ring, the toxicity of PAHs was increase [1]. The benzopyrene is known with strong carcinogenic organic compounds [2], PAHs has intended to be controlled for precedencecontrolled pollutant by U.S. Environmental Protection Agency and the European Union, and 16 kinds of its were regarded as the environmental pollution monitoring parameters [3]. The physical chemical properties of PAHs are stable, when its react, the derivatives are formed by electrophilic substitution reaction. Many environmental factors will affect the reaction of PAHs, such as temperature, light, oxygen, ozone, and other chemical factors, particle surface area adsorbs on PAHs and catalyst, its plays an important role in the process [4].

PAHs is the result of integration of all kinds of pollution emissions in the environment, for identify source of PAHs, qualitative analysis methods mainly include ratio method, profile method and the characteristics of compound method, quantitative analysis methods include chemical mass balance method and multivariate statistical methods (principal component analysis, factor analysis), etc. PAHs are obviously different in composition that it were caused by high temperature combustion or petroleum hydrocarbons, the former is given priority to the female nuclear PAHs by thermal cracking, the latter is rich in alkylation of PAHs, therefore, we commonly used methyl phenanthrene index (the ratio of methyl phenanthrene/fe) to reflect the combustion of PAHs between genesis and petroleum hydrocarbon. Fluoranthene/pyrene (Flu/Pyr) decreased with increasing of atmospheric migration distance, the reason is that fluoranthene is more easily photodegradation than pyrene in the process of atmospheric transmission. In addition, the monomers carbon isotope composition of PAHs can also be used for PAHs pollution migration tracer [5]. Simcik [6] make the quantitative analysis with factor analysis, such as a multiple linear regression method to get the pollution sources of PAHs in Chicago and the Michigan lake area, the results show that coal, natural gas, coke combustion furnace, vehicle emissions contribution respectively is $(48 \pm 5) \%,(26 \pm 2) \%,(14 \pm 3) \%$, and $(9 \pm 4) \%$.

The formation of PAHs can be divided into natural and life sources. Natural sources including volcanic eruptions, forest, grassland natural combustion and biosynthesis. The main source of PAHs is volcanic eruptions, forest, grassland natural combustion in the environment background value. Life sources mainly comes from the incomplete combustion of organic matter, it is the main source of PAHs in the environment [7]. The study show that many hydrocarbons such as coal, oil, wood, organic polymer compounds, and tobacco can produce PAHs in incomplete combustion, when the temperature in $65-900^{\circ} \mathrm{C}$, because lack of oxygen and failed to depth of oxidation, it is easy to PAHs [6].

For the waste gas, waste water and waste dumping, PAHs exert directly pollute on the water, air and soil. PAHs adsorb on the flue gas particles, it is on airflow to the surrounding and faraway, and into the soil and water with the dust, rainfall and snowfall. The PAHs of soil and ground evaporate into the atmosphere by dust again, it is harmful the human and animal through breathing and the food chain.

\section{RESEARCH METHODS AND QUALITY ASSURANCE}

\section{A. Sample collection}

When collect the sample, it must records water sample basic situation, such as GPS, time, temperature, $\mathrm{pH}$, conductivity, color, turbidity, odor, and the surrounding environmental factors such as ecological vegetation and human activities.

In order to ensure the accuracy of the data and comparability, all the samples stored in glass containers, 
before sampling, all glassware must be clean through high temperature baking, after the sample into the laboratory, it should check the list and timely analysis, if not immediately to carry out the analysis work, the sample should be putted in freezer cryopreservation.

\section{B. Sample test}

Laboratory equipment: Analytical balance (0.0001g), soxhlet extractor, thermostatic water bath pot (HHS), condensation reflux pump, rotary evaporation instrument (Heidolph4000, Germany), vacuum pump (SHZ-DIII), ultrasonic vibration meter (SB3200), chromatography column, nitrogen blow dry system, oven, $0.45 \mu \mathrm{m}$ filter, vacuum filter, quantitative filter paper.

Analytical instruments: GC-ECD (Agilent7890A), GCMS (Agilent6890N GC/5975 MSD), all of its are equipped with automatic liquid sampler by Agilent 7683B ALS.

Glass cleaning: All glassware must be cleaned by sulfate-dichromate solution, placed about four hours later, clean it by tap water and distilled water one after another, put into the oven $\left(180^{\circ} \mathrm{C}\right)$ and bake in 4 hours, then put it in a clean place for use.

Reagents: hexane (TEDIA company, U.S, pesticide residues), methylene chloride (TEDIA company, U.S, pesticide residues), acetone (Fisher Scientific company, U.S, HPLC grade); Anhydrous sodium sulfate (Shanghai national medicine corporation, $450^{\circ} \mathrm{Cby}$ the muffle furnace, 6h); Silica gel (Shanghai national medical corporation, chromatography), alumina (Shanghai national medical corporation, chromatography).

Internal standard substance of PAHs is six methyl benzene.

Sample preparation: The pretreatment of water sample adopt method that liquid-liquid extraction (USEPA, 3510B) combined with the chromatographic purification, concentration, solvent replacement operations.

\section{GC analysis}

Testing instrument is Agilent 6980N GC/5975 MSD, sampling system is automatic liquid sampler of Aglient$7683-\mathrm{B}$, chromatographic column is the DB-5MS capillary column $(30.0 \mathrm{~m} \times 0.25 \mathrm{~mm} \times 0.25 \mathrm{~m})$, control temperature according to different temperature program, sample quantity is $2 \mu \mathrm{L}$.

Using chemical workstation software for analysis. The GC-MS workstation software is MSD Chemstation (version number: D. 02.00.275). Using the retention time of various substances in the mixed standard (the characteristics of the ion mass spectrometer contain qualitative), qualitative identification to each material of the sample, quantitative by internal standard method, through quantitative results to correct and calibration. The typical sample as shown in Figure 1.

(3) Quality management and control

In order to ensure the quality of samples produced by environmental monitoring data with scientific, representative and completeness, accuracy, precision and comparability, quality assurance and control involves all the process of management monitoring.

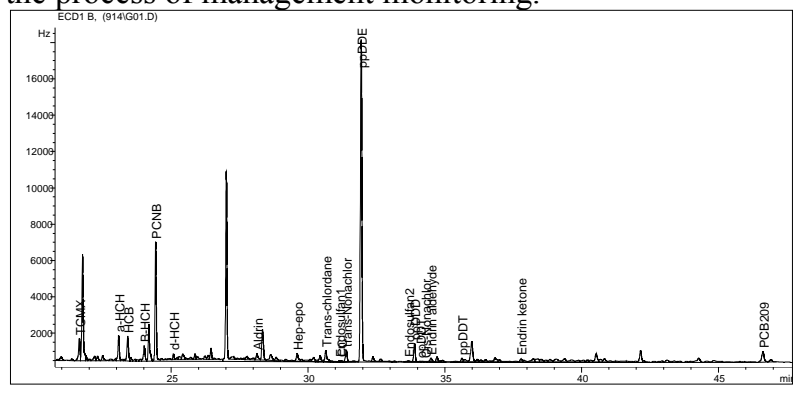

Figure 1. Typical chromatogram of the sample.

\section{Quality assurance and control laboratory analysis (QA/QC)}

Every batch of sample analysis for each project should have parallel blank sample, including the reagent blank and method blank. Quality control personnel select random parallel samples regularly in the laboratory, Pass rate of parallel double determination should be not less than $95 \%$. The range of relative standard deviation (RSD) is $2.6-14.7 \%$.

When something affects the inspection quality, such as water, power, gas, all the samples should be determined again.

When equipment occurs failure, it can determination again by the standby instrument of same level and can satisfy the detection requirements. If no standby instrument, need repair the instrument and test again.

\section{OVERVIEW OF RESEARCH AREA}

This study start since June 2008 to June 2010, it has total seven field investigation, including Guangxi, Chongqing, Yunnan, Guizhou, Guangdong, Hunan and Hubei, it covers basic range of $300 \mathrm{~km}^{2}$ in the southwest karst area, the survey map of study areas is shown in Figure 2.

In this research process, we totally collect 196 water samples, locations is the entrance to the underground river, ponor and export; analysis of organic pollution samples were completed in China university of geosciences (Wuhan), the key laboratory of Biological geology and environmental geology of ministry of education. The field sampling work are shown in Table I.

\section{THE DATA ANALYSIS}

Within the range of four ring PAHs, we divided into light and heavy ring PAHs and discuss the data, its content features are shown in Table II, the content range of light ring is $12.36-12.36 \mathrm{ng} / \mathrm{L}$, average is $115.81 \mathrm{ng} / \mathrm{L}$, the highest point is located at nanshanlaolongdong underground river in Chongqing; the content of light ring is about $40-100 \%$ in total PAHs, average is $94 \%$; the content range of heavy ring PAHs is N.D-140.28ng/L, average is only $7.18 \%$ in study area. 


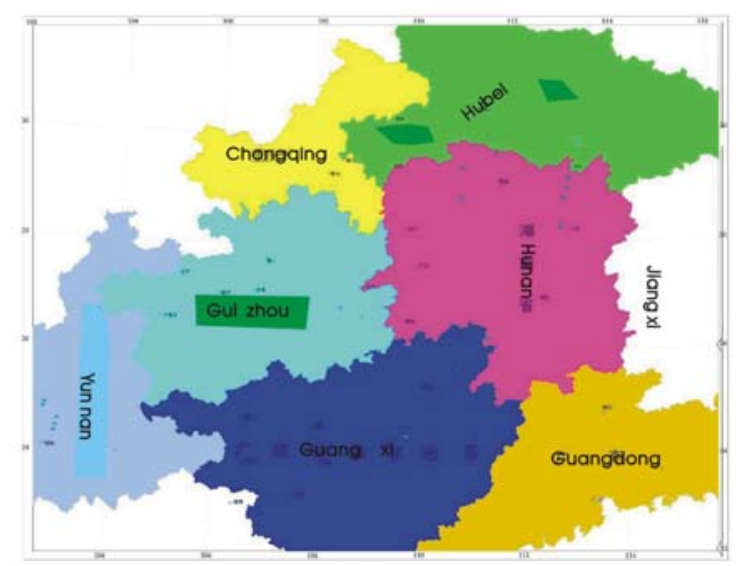

Figure 2. Map of organic pollutants survey in southwestern karst areas

TABLE I. SITUATION OF FIELD SAMPLING.

\begin{tabular}{|l|l|l|}
\hline Area of Work & Sampling Type & Number \\
\hline Guangxi & Water & 30 \\
\hline Guangdong & Water & 9 \\
\hline Yunnan & Water & 15 \\
\hline Guizhou & Water & 5 \\
\hline Chongqing & Water & 125 \\
\hline Hunan and east of Hubei & Water & 13 \\
\hline Total & 196 & \\
\hline
\end{tabular}

TABle II. ANAlysis Results of PAHs Test IN THE Study AREA.

\begin{tabular}{|l|l|l|l|}
\hline $\begin{array}{l}\text { Substances } \\
\text { Name }\end{array}$ & $\begin{array}{l}\text { Range of Content } \\
(\mathrm{ng} / \mathrm{L})\end{array}$ & $\begin{array}{l}\text { Average } \\
(\mathrm{ng} / \mathrm{L})\end{array}$ & $\begin{array}{l}\text { Detection Rate } \\
(\%)\end{array}$ \\
\hline Light PAHs & $12.36-690.89$ & 115.81 & 100 \\
\hline Heavy PAHs & N.D-140.28 & 7.18 & 91 \\
\hline PAHs & $12.36-690.89$ & 122.98 & 100 \\
\hline $\begin{array}{l}\text { Light } \\
\text { PAHs/PAHs }\end{array}$ & $40-100(\%)$ & $94(\%)$ & \\
\hline
\end{tabular}

N.D, Not Detected.

It can be seen that high content of light ring sample more concentrated in the high latitude of northern guizhou to chongqing through geochemical map (Figure 3 and 4), the low latitudes generally characterized by low or not detected; but the distribution of heavy ring content is no so to light ring, the sample of eastern

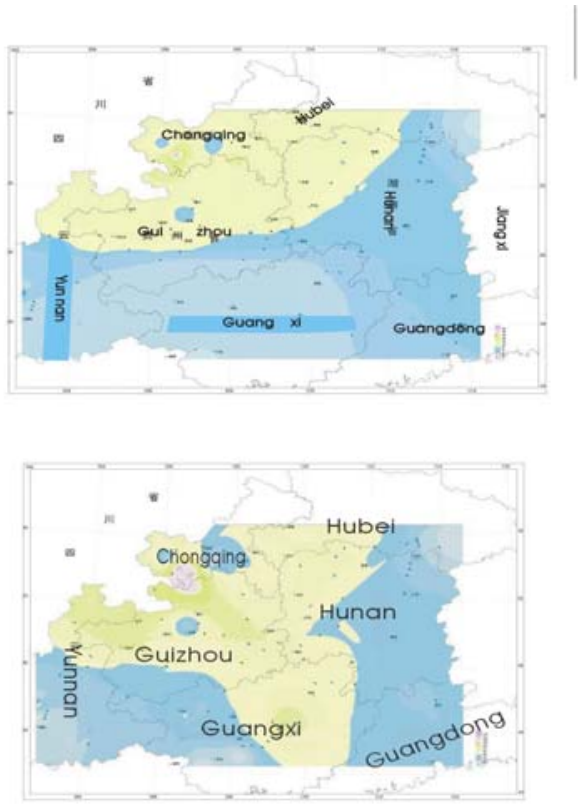

Figure 3. The contour map of light/heavy ring PAHs content.

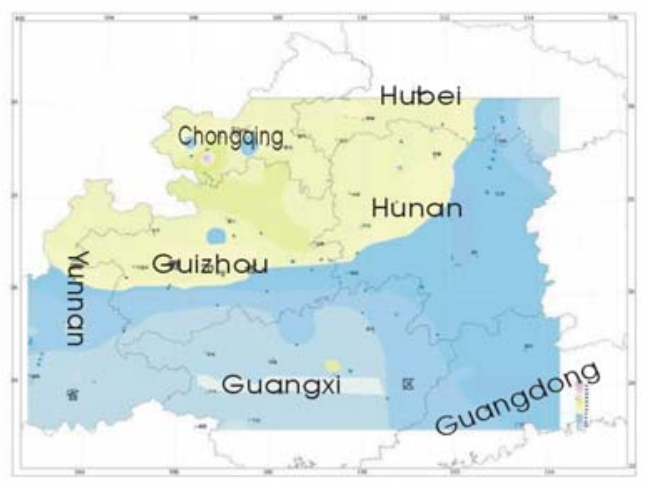

Figure 4. The contour map of total PAHs content.

Guangxi, Hubei, western and northern Chongqing is higher, and the distribution is irregular. The reason is that the of PAHs relate to the molecular weight, the molecular weight is larger, the water solubility is lower, so the content in the water is shown that light ring $>$ heavy ring.

In general, when PAHs of organic is come from combustion in high temperature, the phenanthrene/anthracene ratio is low (less than 10), while it is come from oil sources of PAHs, the ratio is high (more than 15). The ratio of fluorescent anthracene and pyrene also can be used as a judgment: when the ratio greater than 1 , it is come from burning of fossil fuels, otherwise it indicates the input from oil compounds. It is can be seen from the ratio in Table III, $100 \%$ of the sample are less than 10 in water, it illustrated the sources is high temperature combustion of organic matter; the ratio more than 15 only have one sample, it is locate at exports of nanshanlaolongdong underground river in 
Chongqing, it is suggests that the source is likely to be oil compounds. From the ratio of fluorescent anthracene/pyrene, some of them come from combustion of fossil fuels, others from oil compounds of input, and the distribution is irregular. Generally speaking, the main sources of PAHs are come from combustion and oil source mixed in southwest China, belongs to mixed input type.

TABLE III. THE PHENANTHRENE/ANTHRACENE AND FLUORESCENT ANTHRACENE/PYRENE RATIO IN DIFFERENT SAMPLE.

\begin{tabular}{|l|l|l|l|l|}
\hline Type & \multicolumn{2}{|l|}{ Phenanthrene/Anthracene } & \multicolumn{2}{l|}{$\begin{array}{l}\text { Fluorescent } \\
\text { Anthracene/Pyrene }\end{array}$} \\
\hline & Water & $\begin{array}{l}\text { Surface } \\
\text { sediment }\end{array}$ & Water & Surface sediment \\
\hline Range & $0.92-9.91$ & $0.94-15.67$ & $0-6.57$ & $0-2.13$ \\
\hline Average & 1.10 & 2.15 & 1.44 & 1.71 \\
\hline
\end{tabular}

\section{CONCLUSION AND SUGGESTIONS}

(i) We detected the pollutants in all samples in the study area from the data of research. PAHs pollutants is widespread in groundwater in southwest China, because of the special pattern spatial distribution of water and soil resources, the underground river is particularly sensitive to be polluted. Therefore, it is very important that study the distribution of PAHs and migration patterns in groundwater in karst areas southwestern China.

(ii) The migration of PAHs pollutants follow the global distillation model, and the contaminants of high latitudes was more than low latitudes. PAHs have the volatile feature, it makes the migration of atmosphere becomes one of the main mode of transmission, the study areas span contains latitude about $20-35^{\circ}$, Climate type is from high temperature southeastern coastal areas to the hilly area of central plateau, it covering nearly $300 \mathrm{~km}^{2}$, so the study area conform the requirements of the global distillation model migration. From geochemical map can be concluded that PAHs of heavy ring due to the pollution and economic development, road traffic conditions, it is not completely in line with the global model, but PAHs of light ring, are still completely in line with global migration patterns of distillation model.

(iii) The PAHs were mainly comes from agricultural pollution and life pollution in the study area, and artificial source $>$ natural source, the highest points is in northern Chongqing, Guizhou, western region of Hubei and Hunan. It is can be seen from the research data analysis, the life pollution is the most common way of pollution in the study area, it is relationship with rapid economic development in this years, with the improvement of people's living standard, environmental protection consciousness of people was not formed, the relevant laws of government was not perfect, a lot of living garbage and sewage were put into underground rivers, and it make the underground river became the sewer, the pollution was very seriously in nanshan area of Chongqing, the content of PAHs in water is 79.35$675.65 \mathrm{ng} / \mathrm{L}$, surface sediment is $826.16-1508.36 \mathrm{ng} / \mathrm{g}$.

(iv) The landscape belongs to karst in study area, organic environment is very fragile, it is more likely polluted by Persistent Organic Pollutants. The surface soil layer is very thin, rain quickly penetrates into the underground river through fractures, and it cannot through normal form in the atmosphere by evaporation, only can be absorbed by soil, crops and vegetation.

\section{ACKNOWLEDGMENTS}

This study is in the funded by the project that major environmental geological problems in the karst rock mountainous areas in southwest China, under the mutual cooperation between China University of Geosciences and the Chinese Academy of Geological Sciences Institute of Karst Geology.

\section{REFERENCES}

[1] R. Harvey, Polycyclic aromatic hydrocarbons: chemistry and carcinogenicity. In: M.M. Coombs, J. Ashby, M. Hicks, et al. (Eds.), Cambridge Monographs on Cancer Research. Cambridge University Press, Cambridge, 27(6), pp. 1080-1098, 1991.

[2] S.C. Wilson, K.C. JONES, Bioremediation of soil contaminated with polycyclic aromatic hydrocarbons (PAHs). Environmental Pollution, 34(81), pp. 229-249, 1993.

[3] Ketthlh, W.A. Telliard, Priority pollutants: I. A perspective view. Environmental Science and Technology, 13(56), 416-423, 1979.

[4] Keqiang Ding, Yongming Luo, Pahs contaminated soil and bioremediation. Soil, 5(4), pp. 45-56, 2001.

[5] T. Okuda, H. Kumata, H. Kawano, et al., Compound-specific stable carbon isotopic compositions of polycyclic aromatic hydrocarbons in aerosols from China. Extended abstract for the International Conference on Organic Geochemistry, Spain, P-THU, pp. 1-27, 2002.

[6] M.F. Simcik, et al., Source apoortioinment and source/sink relationships of polycyclic aromatic hydrocarbons in the coastal atmosphere of Chicago and Lake Michigan. Atmospheric Environment, 33, pp. 5071-5079, 1999.

[7] Guishan Wang, Zhaoqing Zhong, The harm and the ways to produce of polycyclic aromatic hydrocarbons (PAHs). Shandong Environment, (2), 41, 2001. 